\title{
THE SEPARABLE CLOSURE OF SOME COMMUTATIVE RINGS
}

\author{
BY
}

ANDY R. MAGID( $\left.{ }^{1}\right)$

\begin{abstract}
The separable closure of a commutative ring with an arbitrary number of idempotents is defined and its Galois theory studied. Projective separable algebras over the ring are shown to be determined by the 'Galois groupoid' of the closure. The existence of the closure is demonstrated for certain rings.
\end{abstract}

The finite, separable extension fields of a given field or, more generally, the connected strongly separable algebras over a given connected commutative ring are described by the fundamental theorem of Galois theory applied to the separable closure of the base: the algebras in question correspond to open subgroups of the Galois group of the separable closure. One would like a similar description of separable algebras over an arbitrary base. Since a Galois theory is now available over an arbitrary base [M1], this is equivalent to asking for a separable closure in the general case. Direct generalization from the connected case $[\mathrm{J}, \S 1]$ (that is, requiring the separable closure to be locally strongly separable, to contain no new idempotents, and to contain a homomorphic image of every strongly separable algebra over the base) however, is not possible, as is shown below (3.1). The purpose of the present paper is to find a definition for and in certain cases a construction of the separable closure.

Our definition comes from weakening the first requirement above. We call an algebra quasi-separable if the stalks of the corresponding sheaf over the Boolean spectrum of the base are locally strongly separable. $\$ 2$ below studies such algebras, and their Galois theory is developed. As an illustration, the Galois theory of Boolean rings is discussed. In $\$ 3$, the separable closure is defined as a special maximal element in the set of quasi-separable algebras, and it is shown that the Galois theory of the separable closure determines the strongly separable algebras over the base. Finally, we consider the existence of the separable closure. In general, the separable closure does not exist (see (4.5)

Received by the editors September 24, 1971.

AMS 1970 subject classifications. Primary 13B05; Secondary 13B10, 13 B20.

Key words and phrases. Boolean spectrum, Galois theory, separable algebra, groupoid, profinite space.

(1) Supported by National Science Foundation grant GP-21341A1. 
below). We do demonstrate its existence when the Boolean spectrum of the base is extremally disconnected or countable, and give necessary and sufficient conditions for existence. $\$ 1$ begins with some topological preliminaries, and examples are collected in $\$ 4$.

We will use the definitions and notational conventions of [M1] throughout. In particular, all rings are commutative and algebras faithful. $R$ denotes the generic base ring. $X(R)$ is the Boolean spectrum of $R$. For $x$ in $X(R), R_{x}$ is the stalk of the sheaf over the Boolean spectrum at $x$. For an indepotent $e$ of $R$, $N_{R}(e)=\{x \in X(R): 1-e \in x\}$. If $f: R \rightarrow S$ is a homomorphism, $f^{-1}: X(R) \rightarrow$ $X(S)$ is the associated continuous function. A strongly separable $R$-algebra is a projective $R$-module and a locally strongly separable $R$-algebra is a direct limit of strongly separable subalgebras. A normal $R$-algebra is one which has the same image in the separable closure of $R_{x}$ (for each $x$ in $X(R)$ ) under every homomorphism. A normal, strongly separable algebra is weakly Galois, and locally weakly Galois means the direct limit of such. $C(X, K)$ denotes the continuous functions from $X$ to $K$, and $\operatorname{Aut}_{R}(S)$ is the $R$-algebra automorphisms of $S$. We use "regular" in the sense of von Neumann.

1. The section examines the topological nature of Galois groupoids. We will identify a groupoid with its set of morphisms: a groupoid $G$ is a collection of triples $(a, b, g)$, where $g$ is a morphism in $G$ with range $a$ and domain $b$. A homomorphism of groupoids is a functor. A subgroupoid is a subcategory containing all the identities which is also a groupoid. A topological groupoid is a groupoid in the category of topological spaces (for the definition of a groupoid in an arbitrary category, see [G]). A homomorphism of topological groupoids is a continuous homomorphism. Topological groupoids form a category, which is closed under inverse limits and every finite groupoid (with the discrete topology) belongs to the category.

Let $R, R^{\prime}$ be rings, $S, S^{\prime}$ locally weakly Galois $R$ - and $R^{\prime}$-algebras, and suppose there are ring homomorphisms $b: R \rightarrow R^{\prime}$ and $k: S \rightarrow S^{\prime}$ such that

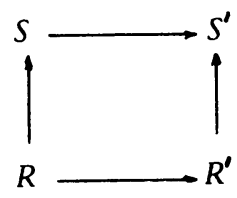

commutes. Let $F: S \otimes_{R} S \rightarrow S^{\prime} \otimes_{R}, S^{\prime}$ be given by $F(x \otimes y)=k(x) \otimes b(y)$.

Proposition 1.1. Let $R, R^{\prime}, S, S^{\prime}, b, k, F$ be as above. Then $F^{-1}$ : $X\left(S^{\prime} \otimes_{R}, S^{\prime}\right) \rightarrow X\left(S \otimes_{R} S\right)$ is a groupoid bomomorphism. If $z^{\prime}=\left(a^{\prime}, b^{\prime}, g^{\prime}\right)$ is in $X\left(S^{\prime} \otimes_{R}, S^{\prime}\right), F^{-1}\left(z^{\prime}\right)=(a, b, g)$ where $a=k^{-1}\left(a^{\prime}\right), b=k^{-1}\left(b^{\prime}\right)$ and $g$ is the unique isomorphism of $S_{b}$ to $S_{a}$ making the following diagram commute: 
(*)

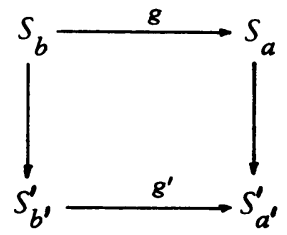

Proof. We begin by showing that the uniqueness in the description of $F^{-1}$ implies that it is a homomorphism. Let $z^{\prime}=\left(a^{\prime}, b^{\prime}, g^{\prime}\right), w^{\prime}=\left(b^{\prime}, c^{\prime}, b^{\prime}\right)$, $F^{-1}\left(z^{\prime}\right)=z=(a, b, g)$ and $F^{-1}\left(w^{\prime}\right)=w=(b, c, b)$. Then

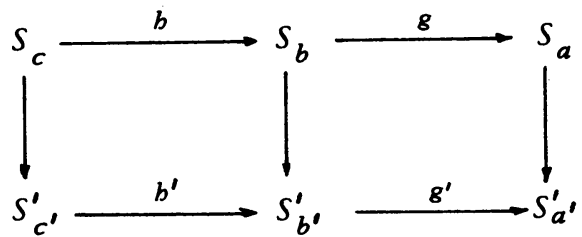

commutes, and hence $F^{-1}\left(z^{\prime} w^{\prime}\right)=F^{-1}\left(a^{\prime}, c^{\prime}, g^{\prime} b^{\prime}\right)=(a, c, g b)=z w$, by uniqueness. We retain the above notation for the rest of the argument. The diagram

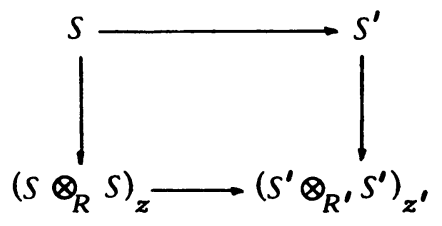

commutes, where the vertical maps are induced by inclusion on the first factor and the lower horizontal map, which we will call $f$, comes from $F$. Clearly $f$ is injective on idempotents since its range and domain are connected. For an idempotent $e$ of $S$, if $1-e$ is in $a,(e \otimes 1)_{z}=1$ and $f\left((e \otimes 1)_{z}\right)=1$. Thus $(k(e) \otimes 1)_{z^{\prime}}=1$ and $1-k(e)$ is in $a^{\prime}$. The argument is reversible so $a=$ $k^{-1}\left(a^{\prime}\right)$. Similarly $b=k^{-1}\left(b^{\prime}\right)$. The diagram

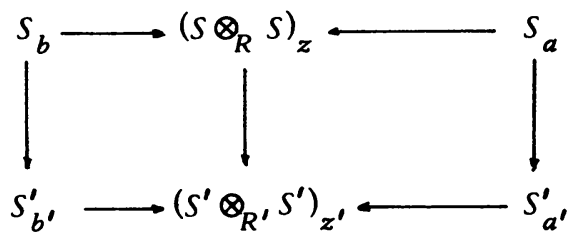

commutes, so that $(*)$ commutes. For uniqueness, suppose that $(*)$ commutes, where $a=k^{-1}\left(a^{\prime}\right)$ and $b=k^{-1}\left(b^{\prime}\right)$. Both vertical maps in (*) are again injective on idempotents. Let $e=\sum s_{i} \otimes t_{i}$ be an idempotent of $S \otimes_{R} S$. If $1-e$ is is in $z, e_{z}=1$ so $\sum\left(s_{i}\right)_{a} g\left(\left(t_{i}\right)_{b}\right)=1$. Applying $k$ and using $(*)$, $\sum k\left(s_{i}\right)_{a}, g^{\prime}\left(k\left(t_{i}\right)_{b}^{\prime}\right)=1$, so $F(e)_{z^{\prime}}=1$ and $1-F(e)$ is in $z^{\prime}$. The argument is reversible, so $z=F^{-1}\left(z^{\prime}\right)$. 
Lemma 1.2. Let $R=\operatorname{dir} \lim R_{i}$ and let $S$ be a weakly Galois $R$-algebra. Then for some $i$ there is a weakly Galois $R_{i}$-algebra $S_{i}$ such that $S=S_{i} \otimes_{R_{i}} R$.

Proof. As in $[E W, 1.2$, p. 235] we can find an $i$ and a strongly separable $R_{i}$-algebra $S_{i}$ such that $S=S_{i} \otimes_{R_{i}} R$. There is a finite subgroup $G$ of Aut $_{R}(\mathrm{~S})$ with $S^{G}=R$ and we can further assume (by increasing $i$, if necessary) that every element of $G$ comes from an $R_{i}$-automorphism of $S_{i}$ by scalar extension. Let $T_{i}=S_{i}^{G}$ and $T=T_{i} \otimes_{R_{i}} R$. Then $T_{i}$ is a strongly separable $R_{i}$-algebra [M2, Theorem 0.9, p. 709] and $S_{i}$ is a weakly Galois $T_{i}$-algebra. Since $S_{i}[G] \rightarrow \operatorname{Hom}_{T}\left(S_{i}, S_{i}\right)$ is surjective $[\mathrm{VZ} 2,3.1, \mathrm{p} .90]$, we have, after tensoring with $R$ over $R_{i}, S[G] \rightarrow \operatorname{Hom}_{T}(S, S)$ is surjective. Thus $\operatorname{Hom}_{T}(S, S)=$ $\operatorname{Hom}_{R}(S, S)$ and, since $T$ is strongly separable over $R, T=R$. Since $T_{i}$ is $R_{i}$-projective, $T_{i}=R_{i}$ and $S_{i}$ is weakly Galois over $R_{i}$.

Theorem 1.3. Let $S$ be a locally weakly Galois $R$-algebra. Then $X\left(S \otimes_{R} S\right)$ is an inverse limit of finite groupoids (i.e. a profinite groupoid).

Proof. First suppose that $S$ is a weakly Galois $R$-algebra. Write $R=$ $\operatorname{dir} \lim R_{i}$, where each $R_{i}$ has finitely many idempotents (e.g., $R_{i}$ is Noetherian). We can assume that the index set has a least element 0 and that, by (1.2), $S=$ $S_{0} \otimes_{R_{0}} R$ for some weakly Galois $R_{0}$-algebra $S_{0}$. Let $S_{i}=S_{0} \otimes_{R_{0}} R_{i} . S_{i}$ is weakly Galois over $R_{i}$ and $S=\operatorname{dir} \lim S_{i}$. It follows that $X\left(S \otimes_{R} S\right) \rightarrow$ proj $\lim X\left(S_{i} \otimes_{R} S_{i}\right)$ is a homeomorphism and, by (1.1), an isomorphism of groupoids. Each $X\left(S_{i}^{i} \otimes_{R} S_{i}\right)$ is finite and discrete, so the inverse limit is a profinite topological groupoid. Now if $S$ is any locally weakly Galois $R$-algebra, $S=\operatorname{dir} \lim S_{i}$, where each $S_{i}$ is weakly Galois. Then $X\left(S \otimes_{R} S\right) \rightarrow$ proj $\lim X\left(S_{i} \otimes_{R} S_{i}\right)$ is a homeomorphism and a groupoid isomorphism. By elementary topology, an inverse limit of profinite groupoids is again profinite.

It is apparently unknown whether a topological groupoid whose underlying space is compact and totally disconnected is a profinite groupoid, although this is the case for groups.

We record for later use some simple facts about profinite groupoids. A weak subgroupoid of a groupoid is a subcategory which is also a subgroupoid.

Proposition 1.4. Let $G$ be a profinite groupoid.

(a) Let $H$ be a closed subgroupoid of G. Then $H$ is the intersection of all open and closed subgroupoids containing $H$.

(b) Let $K$ be a closed weak subgroupoid of $G$. Then $K$ is profinite.

Proof. (a) Given $z$ in $G, z$ not in $H$, there is a finite groupoid $G^{\prime}$ and an onto homomorphism $f: G \rightarrow G^{\prime}$ such that $f(z)$ is not in $f(H)$, since $G$ is pro- 
finite. Then $f^{-1}(f(H))$ is an open and closed subgroupoid of $G$ containing $H$ but not $z$.

(b) If $G=\operatorname{proj} \lim G_{i}$ and $f_{i}: G \rightarrow G_{i}$ is the canonical map, $K=$ proj $\lim f_{i}(K)$.

By (1.4a) and (1.3), the subgroupoids of a Galois groupoid which correspond [M1, 1.10, p. 96] to locally separable algebras are precisely the closed subgroupoids.

The next lemma will be used in the proof of (2.4) below.

Lemma 1.5. Let $E$ be a set of idempotents of $R$ closed under unions, $I$ the ideal of $R$ generated by $E$ and $V(E)=\bigcap\{N(1-e)$ : e $\in E\}$. Then $I=$ $\left\{f\right.$ in $R \mid f_{x}=0$ for all $x$ in $\left.V(E)\right\}$.

Proof. Clearly $I$ is contained in the right-hand side. Suppose $f$ belongs to the right and let $U=\left\{x\right.$ in $\left.X(R) \mid f_{x}=0\right\} . U$ is open and contains $V$, so, by compactness, there are $e_{1}, \cdots, e_{n}$ in $E$ such that $N\left(1-e_{1}\right) \cap \cdots \cap N\left(1-e_{n}\right)$ is contained in $U$. Then if $e=e_{1} \cup \cdots \cup e_{n}, N(1-e)$ is contained in $U$ and $e$ is in $E$. Then $(f(1-e))_{x}=0$ for all $x$ so $f=f e$.

2. In this section we define and study the class of quasi-separable covers.

Definition 2.1. An $R$-algebra $S$ is a quasi-separable cover (of $R$ ) if for each $x$ in $X(R), S_{x}$ is a locally strongly separable $R_{x}$-algebra. $S$ is a separable cover if $S$ is a separable quasi-separable cover. $S$ is a quasi-Galois cover if $S_{x}$ is a locally weakly Galois $R_{x}$-algebra for each $x$. $S$ is a Galois cover if $S$ is a separable quasi-Galois cover.

We make some remarks to help place the above definition in perspective. Clearly locally strongly separable algebras are quasi-separable covers, strongly separable algebras are separable covers, locally weakly Galois algebras are quasi-Galois covers and weakly Galois algebras are Galois covers. In general, all these inclusions are proper, as example (4.1) below shows. There is a quasi-separable cover $S$ of $R$ with $S_{x}$ strongly separable for each $x$ in $X(R)$ but $S$ not a separable cover of $R$, as example (4.2) shows. A separable cover is clearly a flat, faithful, separable algebra.

Proposition 2.2. A separable subalgebra of a quasi-separable cover is a separable cover.

Proof. Let $S$ be the cover of $R, T$ the subalgebra. For each $x$ in $X(R)$, $T_{x}$ is a separable subalgebra of the locally strongly separable $R_{x}$-algebra $S_{x}$ and hence, by $[\mathrm{M} 1,1.2$, p. 90], strongly separable.

Proposition 2.3a. Let $S$ be an R-algebra, $T$ a subalgebra. 
(i) If $T$ is a separable cover of $R$ and $S$ a separable cover of $T$, then $S$ is a separable cover of $R$.

(ii) If $S$ is a separable cover of $R$ and $T$ a quasi-separable cover of $R$ then $S$ is a separable cover of $T$.

Proof. We may assume that $R$ is connected. Then part (i) is standard. For (ii), we observe that since, by Galois theory over connected rings [VZ1, p. 731], there are only finitely many separable subalgebras of $S$ and hence $T$ is in fact separable. Then $S$ is strongly separable over $T$ and the result follows.

As example (4.2) shows, even if, in the notation of (2.3ii), $S$ is strongly separable over $R$ and $T$ is locally strongly separable, $S$ need not be strongly separable over $T$, although it is a separable cover of $T$. Thus separable covers arise in a natural way in the study of locally strongly separable algebras.

Proposition 2.3. A quasi-separable cover is a separable cover if and only if it is finitely generated as an $R$-module.

Proof. A finitely generated algebra which is separable at each localization of the base is separable [B, 2.10, p. 94], so a finitely generated quasi-separable cover is a separable cover. For the converse, let $e=\sum a_{i} \otimes b_{i}$ be a separability idempotent for $S$. For each $x$ in $X(R), S_{x}$ is a strongly separable $R_{x}$-algebra with separability idempotent $e_{x}$. By the proof of [DI, 2.1, p. 92], $\left(a_{1}\right)_{x}, \cdots$, $\left(a_{n}\right)_{x}$ generate $S_{x}$ as an $R_{x}$-module. Since this holds for each $x, S$ is generated by $a_{1}, \cdots, a_{n}$.

Lemma 2.4. Let $T$ be a locally strongly separable $R$-aglebra, $S$ a quasiseparable cover of $R$ and $T \rightarrow S$ a surjection with kernel $I$. Then $I$ is generated by idempotents.

Proof. With $T=\operatorname{dir} \lim T_{i}$ with each $T_{i}$ strongly separable, let $I_{i}=$ $I \cap T_{i}$. It suffices to show that each $I_{i}$ is generated by idempotents and hence we can assume that $T$ is strongly separable. $I_{x}$ is the kernel of $T_{x} \rightarrow S_{x}$ for each $x$ in $X(R)$, and by $[\mathrm{J}, 1.6, \mathrm{p} .464] I_{x}$ is generated by an idempotent. This idempotent can be lifted to an idempotent in $I$, and thus there is a set $E$ of idempotents of $I$ such that $E_{x}$ generates $I_{x}$ for each $x$, so $E$ generates $I$.

We can now describe the structure of quasi-separable covers.

Theorem 2.5. Let $S$ be a locally strongly separable (locally weakly Galois) $R$-algebra and $I$ an ideal of $S$ generated by idempotents sucb that $I \cap R=0$. Then $S / I$ is a quasi-separable (quasi-Galois) cover of $R$; conversely, every quasi-separable (quasi-Galois) cover is of this form.

Proof. Since for $x$ in $X(R),(\mathrm{S} / I)_{x}=S_{x} / I_{x}$ and $I_{x}$ is generated by idempo- 
tents, it suffices to prove the first part in the case where $R$ is connected. Clearly $S / I$ is locally separable (and if $S$ is locally weakly Galois, also normal in the sense of [M1, p. 92]); we show it to be locally strongly separable by embedding it in a locally strongly separable $R$-algebra and applying [M1, 1.2, p. 90]. Let $W$ be a separable closure of $R$ and $T=S \otimes_{R} W . T$ is a locally strongly separable $S$-algebra. $T$ is faithfully $S$-flat $[M 1,1.1$, p. 90], and hence $T I \cap S=I$, so $S / I \rightarrow T / T I$ is injective. $T$ is a locally weakly Galois $W$-algebra; since $W$ is separably closed, by $[M 1,3.7$, p. 99] $T$ is isomorphic to $C(X, W)$ for some profinite space $X$. Let $E$ be the set of idempotents in $T I$. We have $T E=T I$. Let $V(E)$ be as in (1.5). $V(E)$ is a closed subset of $X$ and by (1.5) again the surjection $C(X, W) \rightarrow C(V(E), W)$ has kernel $T E$. Thus $T / T I$ is isomorphic to $C(V(E), W)$ which is locally strongly separable over $R$.

Now suppose $T$ is any quasi-separable (quasi-Galois) cover of $R$. For each $x$ in $X(R)$ and $a$ in $T_{x}$ we can, using the techniques of [M3, $\left.\$ 1\right]$, find a strongly separable (weakly Galois) $R$-algebra $S(a, x)$ and a homomorphism $f$ : $S(a, x) \rightarrow T$ such that $a$ is in the image of $f_{x}$. Let $S=\bigotimes S(a, x)$, where $(a, x)$ ranges over all pairs as above. $S$ is locally strongly separable (locally weakly Galois) and we have constructed a surjection $S \rightarrow T$. By (2.4), the kernel $I$ of this map is generated by idempotents and $I \cap R=0$.

Corollary 2.6. Let $S$ be a strongly separable (weakly Galois) $R$-algebra and $I$ an ideal of $S$ generated by idempotents such that $I \cap R=0$. Then $S / I$ is a separable (Galois) cover of $R$; conversely, every separable (Galois) cover is of this form.

Corollary 2.7. Every quasi-separable (quasi-Galois) cover is a direct limit of subalgebras which are separable (Galois) covers.

Proof. By (2.5) there is a locally strongly separable (locally weakly Galois) algebra $S$ mapping onto the cover $T . S=\operatorname{dir} \lim S_{i}$ where each $S_{i}$ is strongly separable (weakly Galois). If $T_{i}$ is the image of $S_{i}$ in $T, T_{i}$ is a cover of the proper type and $T=\operatorname{dir} \lim T_{i}$.

We recall that an $R$-algebra $S$ is locally connected if for each $x$ in $X(R)$, $S_{x}$ is connected (equivalently, every idempotent of $S$ is in $R$ ). For $R$-algebras $S$ and $T, \operatorname{Alg}_{R}(S, T)$ is the set of $R$-algebra homomorphisms from $S$ to $T$.

Lemma 2.7. Let $S$ be a locally connected separable cover of $R$ generated as an $R$-module by $n$ elements and let $T$ be a locally connected R-algebra. Then Card $\left(\operatorname{Alg}_{R}(S, T)\right) \leq n$. Card $(X(R))$.

Proof. If $f$ and $g$ are in $\operatorname{Alg}_{R}(S, T)$ and $f_{x}=g_{x}$ for all $x$ in $X(R)$ then $f=g$. Thus $\operatorname{Alg}_{R}(S, T) \rightarrow \Pi_{x} A_{\lg _{R_{x}}}\left(S_{x}, T_{x}\right)$ is injective. By [J, 1.3, P. 462], 
Card $\left(\operatorname{Alg}_{R_{x}}\left(S_{x}, T_{x}\right)\right)$ is at most $n$, and the result follows.

Corollary 2.9. The cardinalities of quasi-separable locally connected $R$ algebras are bounded.

Proof. By (2.7), any such algebra is a direct limit of subalgebras which are locally connected separable covers; there is only a set of such and, by (2.8), only a bounded number of ways each can be embedded in the algebra.

We will use (2.9) in the construction of the separable closure below. We conclude this section with a statement of the Galois theory of quasi-Galois covers. Since the arguments are almost identical with $[M 1, \S 1]$, we shall omit proofs. Let $S$ be a quasi-Galois $R$-algebra. We make $X\left(S \otimes_{R} S\right)$ into a groupoid as follows: Let $f: X\left(S \otimes_{R} S\right) \rightarrow X(R)$. be the canonical map, and let $x$ be in $X(R)$. Then $f^{-1}(x)=X\left(S_{x} \otimes_{R} S_{x}\right)$ and this latter is a groupoid by $[M 1,1.8$, p. 93]. Thus $X\left(S \otimes_{R} S\right)$ becomes a groupoid with compositions allowed only between members of the same fibre over $X(R)$. Using (2.5), find a locally weakly Galois $R$-algebra $T$ and a surjection $T \rightarrow S$. The groupoid structure on $X\left(T \otimes_{R} T\right)$ is also fibre-wise, and hence (1.1), applied to the fibres over points of $X(R)$ of the induced map $g: X\left(S \otimes_{R} S\right) \rightarrow X\left(T \otimes_{R} T\right)$ shows that $g$ is a groupoid homomorphism; since $g$ is injective, it follows that $X\left(S \otimes_{R} S\right)$ is iso-. morphic and homeomorphic to a closed weak subgroupoid of $X\left(T \otimes_{R} T\right)$. We conclude by (1.4b) that $X\left(S \otimes_{R} S\right)$ is a profinite groupoid. Now by repeating the arguments of $[\mathrm{M} 1]$, we have

Theorem 2.10. Let $S$ be a quasi-Galois cover of $R$. Then $X\left(S \otimes_{R} S\right)$ is a topological groupoid and there is a one-one correspondence between the quasiseparable covers of $R$ in $S$ and closed subgroupoids of $X\left(S \otimes_{R} S\right)$. Separable covers correspond to open-and-closed subgroupoids.

We will illustrate $(2.10)$ in the case of Boolean rings..

Lemma 2.11. Let $R$ be a regular ring such that for every $x$ in $X(R), R_{x}$ is a perfect field. Then an integral, reduced extension $S$ of $R$ is a regular ring and a quasi-separable cover, and conversely.

Proof. For all $x$ in $X(R), S_{x}$ is a reduced integral extension of the perfect field $R_{x}$, so we may assume that $R=R_{x}$. But then $S$ is a direct limit of finite products of finite separable field extensions of $R$, and hence regular and locally strongly separable. Conversely, a quasi-separable cover of a field is a direct limit of finite products of finite separable field extensions, and the result follows.

Corollary 2.12. Let $S$ be a Boolean ring, $R$ a subring. Then $S$ is a Galois cover of $R$. 
Proof. By (2.11), $S$ is a quasi-separable cover of $R$. For any $x$ in $X(R)$, any image of $S_{x}$ in a separable closure of $R_{x}$ is $Z / 2 Z$, so $S$ is normal over $R$.

We continue the notation of (2.12). By the Stone representation theorem, $R=C(Y, Z / 2 Z)$ and $S=C(X, Z / 2 Z)$ for suitable compact, zero-dimensional spaces $X$ and $Y$ and the inclusion of $R$ into $S$ induces a surjection $X \rightarrow Y$. Again by the Stone theorem, rings between $R$ and $S$ correspond to spaces covered by $X$ and covering $Y$, and, since these spaces are quotients of $X$, the spaces correspond to closed equivalence relations on $X$ finer than the equivalence relation whose quotient is $Y$. In other terms, these are the closed equivalence relations contained in $X \times_{Y} X . X \times_{Y} X$ can be given the structure of a groupoid by defining $(a, b)(b, c)=(a, c)$, and then the closed subgroupoids are the closed equivalence relations. On the other hand, $X \times_{Y} X=X\left(S \otimes_{R} S\right)$, and the groupoid structure defined by $(2.10)$ is the same as that given here.

We now show that this Galois theory of Boolean rings cannot be deduced from that of [M1], that is, that Boolean ring extensions need not be locally strongly separable. We will work, however, in more generality.

Lemma 2.13. Let $S$ be an R-algebra, e an idempotent of $S$. Then $R+R e$ is a separable $R$-algebra.

Proof. Let $T=R[x] /\left(x^{2}-x\right)$, and let $f=x+\left(x^{2}-x\right)$. Then $f^{2}=f$ and there are homomorphisms of $T$ into $R$ sending $f$ to 0 and 1 respectively. $T=$ $T f \times T(1-f)$ and $T f=R \dot{f}$ and $T(1-f)=R(1-f)$. If $r f=0$ for $r$ in $R$, then sending $f$ to 1 shows that $r=0$, and hence $R f$ is isomorphic to $R$. In a similar fashion, $R(1-f)$ is isomorphic to $R$. Thus $T$ is isomorphic to $R \times R$ and hence separable. $R+R e$ is a homomorphic image of $T$ and so also is separable.

Proposition 2.14. Let $S$ be a strongly separable R-algebra. Then the induced map $p: X(S) \rightarrow X(R)$ is open.

Proof. Let $e$ be an idempotent of $S$. Then $T=R+R e$ is a separable $R$ algebra (2.13) and hence also strongly separable. $T e=R e$ is a direct summand of $T$ and hence also a projective $R$-module, so $\{r$ in $R: r e=0\}$ is a direct summand of $R$, say equal to $R(1-f)$ where $f$ is an idempotent of $R$. We first have $N_{S}(e)$ contained in $p^{-1}\left(N_{R}(f)\right)$, since $e(1-f)=0$, so for $x$ in $x(S)$, e not in $x$ implies that $1-f$ is in $x \cap R$. Also $e=e f$, so we can replace $R$ by $R f$ and assume that $R e$ is a faithful $R$-module. We then claim that $p\left(N_{S}(e)\right)=X(R)$, for the hypotheses remain when we pass from $R$ to $R_{x}$ for each $x$ in $X(R)$ (in particular, $e_{x}$ is not zero), and we may assume $R=R_{x}$. But then $N_{S}(e)$ is nonempty and $X(R)$ is a single point, so $p$ is surjective. Thus, returning to our original $R$, we have $p\left(N_{S}(e)\right)=N_{R}(f)$. 
The converse of (2.14) is, in general, false, so example (4.3) illustrates. We will establish a partial converse, however.

Lemma 2.15. Let $S$ be a separable cover of $R$ generated over $R$ by a finite set of idempotents. Then $S$ is strongly separable over $R$ if and only if the induced map $p: X(S) \rightarrow X(R)$ is open.

Proof. Suppose $p$ is open. Let $e$ be an idempotent of $S$. Since $N_{S}(e)$ is compact and open, so is $p^{\prime}\left(N_{S}(e)\right)$, so $p\left(N_{S}(e)\right)=N_{R}(f)$ for some idempotent $f$ of $R$. Also, $p\left(N_{S}(e)\right)=\left\{x\right.$ in $X(R)$ : there is $y$ in $X(S)$ with $e_{y}=1$ and $\left.y \cap R=x\right\}=$ $\left\{x\right.$ in $\left.X(R): e_{x} \neq 0\right\}$. A similar argument applied to $1-e$ shows that $\{x$ in $\left.X(R): e_{x} \neq 1\right\}$ is also open and closed in $X(R)$. Let $U=\left\{x\right.$ in $X(R): e_{x}$ is in $\left.R_{x}\right\}, V=X(R)-U$. By the above, $U$ is open and closed. Let $T=R+R e$. By (2.13), $T$ is a separable $R$-algebra and thus, by (2.2), quasi-separable. For $x$ in $X(R),\left[T_{x}: R_{x}\right]=1$ or 2 , the first happening if $x$ is in $U$, the second if $x$ is in $V$. Thus the rank of $T$ is locally constant and hence $T$ is strongly separable over $R$. The converse follows from (2.14).

Following DeMeyer [D], we will call a finite product of rings of functions from compact, 0-dimensional spaces to connected rings a weakly uniform ring. A Boolean ring is, of course, weakly uniform.

Proposition 2.16. A strongly separable extension $S$ of a weakly uniform ring $R$ is weakly uniform.

Proof. We can suppose $R=C(X, K)$, where $K$ is connected, $X$ compact and 0-dimensional. Suppose $x$ is in $X(=X(R))$ and $S_{x}=F_{1} \times \cdots \times F_{n}, F_{i}$ a connected, strongly separable $K$-algebra. Let $T=C\left(X, F_{1} \times \cdots \times F_{n}\right)=\Pi C\left(X, F_{i}\right)$. The $R$-algebras $T$ and $S$ are both finitely generated and projective as $R$-modules, and isomorphic at $x$. Thus, as in [M3], T and $S$ are isomorphic on a neighborhood of $x$, and on this neighborhood $S$ is weakly uniform. Since $X$ is covered by such neighborhoods, $S$ is weakly uniform.

Proposition 2.17. Let $R$ be a weakly uniform ring, $S$ a separable cover of $R$. Then $S$ is strongly separable if and only if the induced map $p: X(S) \rightarrow X(R)$ is open.

Proof. We can suppose $R=C(X, K), K$ connected, $X$ 0-dimensional. Let $x$ be in $X$, and define $F_{i}, T$ as in the proof of (2.16). The isomorphism of $S_{x}$ to $T_{x}$ lifts to a homomorphism $T \rightarrow S$. Since $S$ is finitely generated (2.3), $T$ is surjective on a neighborhood of $x$. For each $y$ in this neighborhood, $S_{y}$ is a product of some of the $F_{i} . X$ is covered by a finite number of such neighborhoods. Thus there is a connected, finitely generated Galois extension $L$ of $K$ such that for every $x$ in $X, S_{x}$ is a product of subalgebras of $L$. Let $W=$ 
$S \otimes_{K} L . W$ is strongly separable over $S$ so that $X(W) \rightarrow X(S)$ is open (2.14) and hence $X(W) \rightarrow X(R)$ is open. $W$ is a separable $R$-algebra; if it is strongly separable then so is the subalgebra $S$. Thus we may assume $W=S$. For every $x$ in $X$, then, $S_{x}$ is a product of copies of $L$ and so for every $y$ in $X(S), S_{y}=L$, and $S=C(X(S), L)$. Let $V=C(X, L) . V$ is between $R$ and $S$, and $V$ is strongly separable over $R . S$ is generated over $V$ by idempotents and hence, by (2.13), strongly separable over $V$ and therefore over $R$. The converse implication is (2.14).

Corollary 2.18. Let $S$ be a Boolean ring, $R$ a subring. $S$ is locally strongly separable over $R$ if and only if $X(S)$ is an inverse limit of spaces mapping to $X(R)$ by compatible open sujections.

Proof. If $S$ is locally strongly separable over $R, S=\operatorname{dir} \lim S_{i}$, where $S_{i}$ is strongly separable over $R$, and $X(S)=\operatorname{proj} \lim X\left(S_{i}\right)$. By (2.14), each $X\left(S_{i}\right)$ is an open covering of $X(R)$. The converse follows similarly using (2.17).

Example (4.1) shows that the condition of (2.18) is not always satisfied. Thus to encompass the Galois theory of Boolean rings (which, as shown above, depends only on the Stone representation theorem) in the Galois theory of separable algebras, it is necessary to go to separable covers; strongly separable algebras alone are insufficient.

3. In this section we define and construct separable closures. Because of Example (4.4), we shall not require the separable closure to be locally strongly separable. However, we would like our definition to agree with that of $[\mathrm{J}]$ when the base ring has no nontrivial idempotents. This leads to

Definition 3.1. A separable closure of $R$ is a locally connected quasiseparable cover of $R$ which receives a homomorphism from every strongly separable $R$-algebra.

Proposition 3.2. Suppose $R$ is connected. Then $S$ is a separable closure of $R$ if and only if $S$ is a connected locally strongly separable $R$-algebra which has no proper, connected strongly separable extensions.

Proof. Suppose $S$ is a separable closure of $R$. As in [J, 1.4, p. 463] there is a locally strongly separable $R$-algebra $S_{0}$ which contains $S$ and has no proper strongly separable extensions. $S_{0}=\operatorname{dir} \lim S_{i}$ where each $S_{i}$ is strongly separable. Thus $\operatorname{Alg}_{R}\left(S_{0}, S\right)=$ proj $\lim \operatorname{Alg}_{R}\left(S_{i}, S\right)$ and the latter is nonempty, being an inverse limit of nonempty finite sets. Choose a homomorphism from $S_{0}$ to $S$. Composed with the inclusion, it gives an endomorphism of $S_{0}$, hence [J, 1.7, p. 465] an automorphism. Thus $S=S_{0}$. The converse is found in [J, p. 464].

(3.2) shows that (3.1) reduces to [J, Definition 3, p. 463] when the base is connected. 
Proposition 3.3. $S$ is a separable closure of $R$ if and only if for every $x$ in $X(R), S_{x}$ is a separable closure of $R_{x}$.

Proof. $S$ is locally connected and quasi-se parable if and only if for each $x$, $S_{x}$ is connected and locally strongly separable. If $S$ is a separable closure, $x$ in $X(R)$, and $T_{0}$ is a strongly-separable $R_{x}$-algebra, we can find [M3, 1.6, p. 116] a strongly separable $R$-algebra $T$ such that $T_{x}=T_{0}$. There is an $b: T \rightarrow S$, so $b_{x}: T_{0} \rightarrow S_{x}$, and $S_{x}$ is a separable closure. If each $S_{x}$ is a separable closure and $T$ a strongly separable $R$-algebra there is, for each $x$, some $f_{x}: T_{x} \rightarrow S_{x}$. Again as in [M3], these $f_{x}$ 's can be pieced together to give an $f: T \rightarrow S$. Thus $S$ is a separable closure.

Corollary 3.4. A separable closure is a quasi-Galois cover.

Proof. This follows from (3.3), (3.2), and [J, Remark 1, p. 466].

Using (3.3), we can give examples of separable closures. If $R$ is a connected ring with separable closure $S, C(X, S)$ is the separable closure of $C(X, R)$ for every 0 -dimensional space $X$.

We now show that the Galois theory of a separable closure of $R$ determines the strongly separable $R$-algebras. If $S$ is a separable closure of $R$, the groupoid $X\left(S \otimes_{R} S\right)$ of (2.10) has the property that the fibre over the point $x$ of $X(R)$ is $X\left(S_{x} \otimes_{R_{x}} S_{x}\right)=$ Aut $R_{x}\left(S_{x}\right)$. Thus every morphism in $X\left(S \otimes_{R} S\right)$ is an automorphism, and $X(R)$ is the set of objects of $X\left(S \otimes_{R} S\right)$.

Recall that a category is skeletal if every isomorphism is an automorphism.

Definition 3.5. Let $G$ be a skeletal groupoid with set of objects $X$. A G-set is a set $M$ with maps $p: M \rightarrow X$ and $k: G \times_{X} M \rightarrow M$ such that for $x$ in $X, g$, $b$ in $\operatorname{hom}_{G}(x, x)$ and $a$ in $p^{-1}(x)$ we have

(i) $k(1, a)=a \quad(1=$ identity on $x)$,

(ii) $k(g, k(b, a))=k(g h, a)$.

We will write $g a$ for $k(g, a)$. Note that each fibre $p^{-1}(x)$ is a $\operatorname{hom}_{G}(x, x)$ set in the usual sense of group action on a set.

Let $S$ be a separable closure of $R$ and $T$ a strongly separable $R$-algebra. We make $X\left(S \otimes_{R} T\right)$ an $X\left(S \otimes_{R} S\right)$-set: the inclusion of $R$ in $S \otimes_{R} T$ induces $p: X\left(S \otimes_{R} T\right) \rightarrow X(R)$; we define $k$ componentwise. For this we can assume $R$ is connected, and then $X\left(S \otimes_{R} T\right)=\operatorname{Alg}_{R}(T, S)$ and $X\left(S \otimes_{R} S\right)=$ Aut $_{R}(S)$. For $a$ in the former and $g$ in the latter we define $k(g, a)=g a$. Under these definitions, we call $X\left(S \otimes_{R} T\right)$ the Galois set corresponding to $T$.

Theorem 3.6. Let $R$ bave a separable closure $S$. Then a strongly separable $R$-algebra is determined by its corresponding Galois set.

Proof. Let $T$ and $T^{\prime}$ be strongly separable $R$-algebras. $T$ is isomorphic to 
$T^{\prime}$ if and only if for each $x$ in $X(R), T_{x}$ is isomorphic to $T_{x}^{\prime}$. Thus we can work componentwise and assume $R$ is connected. Here the argument is standard: $T=T_{1} \times \cdots \times T_{n}$ where $T_{i}$ is connected, so $\operatorname{Alg}_{R}(T, S)=\bigcup \operatorname{Alg}_{R}\left(T_{i}, S\right)$ as Aut $_{R}(S)$-sets. Since $T_{i}$ is connected, we may assume it is a subalgebra of $S$. $\operatorname{Alg}_{R}\left(T_{i}, S\right)$ is a transitive $A u t{ }_{R}(S)$-set, and thus the stabilizer of the inclusion $T_{i} \subset S$ is determined up to conjugation. But this stabilizer is the subgroup of Aut ${ }_{R}(S)$ leaving $T_{i}$ fixed, and which determines $T_{i}$ by the fundamental theorem of Galois theory. Thus $T_{i}$ is determined up to isomorphism by the Galois set $\operatorname{Alg}_{R}\left(T_{i}, S\right)$, and hence $T$ is determined by its Galois set.

We note that (3.6), which is the basic result to which any definition of separable closure should lead, follows immediately from the known the ory in the connected case, since all data involved localize properly with respect to the Boolean spectrum. This is the main reas on for the requirement that the separable closure be locally connected.

Proposition 3.7. A separable closure bas no proper, locally connected separable covers.

Proof. If $T$ is a locally connected separable cover of the separable closure $S$, for each $x$ in $X(R), T_{x}$ is a strongly separable connected $S_{x}$-algebra hence, by (3.3) and (3.4), equal to $S_{x}$. Thus $T=S$.

(3.7) suggests a possible construction of the separable closure. We call a quasi-separable locally connected cover a maximal cover if it has no proper locally connected separable covers.

Proposition 3.8. Maximal covers exist.

Proof. Since the cardinalities of locally connected quasi-separable covers are bounded (2.9), the idea of the proof of [J, 1.4, p. 463] works here.

A maximal cover $S$ will be a separable closure if it contains homomorphic images of strongly separable algebras, i.e. if it satisfies:

(+) If $T$ is a strongly separable $R$-algebra, there exists an ideal $I$ of $S \otimes_{R} T$, generated by idempotents, such that $I \cap S=0$ and $S \otimes_{R} T /$ is a locally connected $S$-algebra.

If $(+)$ obtains, then since $S \otimes_{R} T / I$ is locally connected and, by (2.5), a separable cover of $S$, it must equal $S$, and hence the composite $T \rightarrow S \otimes_{R} T \rightarrow S \otimes_{R}$ $T / I=S$ is the necessary homomorphism. Since ideals of a ring generated by idempotents correspond to closed subsets of the Boolean spectrum of the ring [P, 9.3, p. 37], we can translate $(+)$ to

$(++)$ If $T$ is a strongly separable $R$-algebra the canonical surjection $X\left(S \otimes_{R} T\right) \rightarrow X(S)(=X(R))$ has a continuous section. 
(In making the translation, one goes from $(+)$ to $(++)$ by taking $X(S)=$ $X\left(S \otimes_{R} T / I\right) \rightarrow X\left(S \otimes_{R} T\right)$ for the section and from $(++)$ to $(+)$ by taking for $I$ the ideal corresponding to the image of the section.) As an immediate consequence of $(++)$ we have

Corollary 3.9. If $X(R)$ is an extremally disconnected space, $R$ bas a separable closure.

Proof. By [W, Example 8, p. 311], any surjection $Y \rightarrow X(R)$ admits a section, for $Y$ any compact Hausdorff space.

Thus if $X(R)$ is the Stone- $\breve{C}$ ech compactification of a discrete space, $R$ has a separable closure. This happens if $R$ is a product of connected rings, or if $R$ is the ring of adeles in a number field.

If $X$ is the space of Example 4.1, not every surjection $Y \rightarrow X$ admits a section for arbitrary spaces $Y$. However, we do have

Proposition 3.10. Suppose that $X(R)$ is countable. Then $R$ bas a separable closure.

Proof. We will show the following: Let $X=\left\{x_{1}, x_{2}, \ldots\right\}$ and let $T$ be a weakly Galois $R$-algebra. For each $n$ there is a weakly Galois $R$-algebra $T_{n}$ and a surjection $T \rightarrow T_{n}$ such that $\left(T_{n}\right)_{x_{n}}$ is connected. We will construct the algebras inductively so that $T_{n} \rightarrow T_{n+1}$ and hence $T_{0}=\operatorname{dir} \lim T_{n}$ will be a locally connected image of $T$. Then $(+)$ implies the result. Clearly, it will suffice to construct $T_{1}$. If $e$ is an idempotent of $T$ such that $e_{x} \neq 0$ for any $x$ while $(e)_{x_{1}}$ is a minimal idempotent then $T /(1-e) T=T_{1}$ will work. We proceed to find such an $e$. Let $G$ be a finite group such that $T^{G}=R$. Let $e_{1}$ be a minimal idempotent of $(T)_{x_{1}}$. Choose $g_{1}, \cdots, g_{k}$ in $G$ such that $g_{1}\left(e_{1}\right)+\cdots+g_{k}\left(e_{1}\right)=1_{x_{1}}$ (where $\left.g_{i}\left(e_{1}\right)=\left(g_{i}\right)_{x_{1}}\left(e_{1}\right)\right)$; this is possible since, by [VZ1, p. 724], $G_{x_{1}}$ acts transitively on idempotents. We can find an idempotent $e_{0}$ of $S$ which goes to $e_{1}$ at $x_{1}$ and such that $\left(g_{1}\left(e_{0}\right)+\cdots+g_{k}\left(e_{0}\right)\right)_{x}=1_{x}$ for all $x$ in an open-closed neighborhood of $x_{1}$. In particular, $\left(e_{0}\right)_{x} \neq 0$ in that neighborhood. We take $e$ equal to $e_{0}$ on the neighborhood and equal to 1 off it.

(3.9) and (3.10) should, of course, only be special cases of a general result describing when the separable closure exists. What this general result is remains an open question.

4. We have collected in this section examples referred to in the other sections. We will make the following conventions: let $X$ be the subspace $\{1,1 / 2,1 / 3, \ldots, 0\}$ of the unit interval; let $Q$ denote the rationals, $R$ the reals and $C$ the complexes (all three with the discrete topology).

Example 4.1. Let $W=X \times\{0,1\}$ and let $Y$ be the closed subspace 
$\{(1 / k) \mid k$ even $\} \cup\{(1 / k, 0): k$ odd $\} \cup\{(0,0),(0,1)\}$. Map $W$ (and hence $Y$ ) to $X$ by projection on the first factor. Let $D=C(X, Z / 2 Z), E=(Y, Z / 2 Z)$ and $F=$ $C(W, Z / 2 Z)$. All three are Boolean rings. $D$ is a subring of $E$ and $F$ and $F$ maps surjectively to $E$. $F$ is a finitely generated $D$ module, hence so is $E$. The map of $Y$ to $X$ is not open $((X \times\{1\}) \cap Y$ does not map to an open set $)$ so by (2.18), $E$ is not a strongly separable $D$-algebra. By (2.12), however, $E$ is a Galois cover of $D$.

Example 4.2. Let $D=C(X, R), E=C(X, C)$ and $F=\{f \in E: f(0) \in R\}$. Then $X(D)=X(F)=X$ and for $x$ in $X, D_{x}=E_{x}=F_{x}=C$ for $x \neq 0$, while $D_{0}=F_{0}=$ $R$ and $E_{0}=C . E$ is a weakly Galois $D$-algebra. $F$ is a locally strongly separable $D$-algebra such that $F_{x}$ is separable for each $x$ but $F$ is not a separable $D$-algebra, since it is not finitely generated over $D . E$ is a separable cover of $F$ by (2.11) but not a strongly separable algebra since its rank is discontinuous.

Example 4.3. Let $Y=\{(x, x): x \in X\} \cup X \times\{0\}$. Map $Y$ to $X$ by projection on the first factor. Let $D=C(X, Z / 2 Z), E=C(Y, Z / 2 Z)$. For $x$ in $X, E_{x}=$ $(Z / 2 Z)^{(2)}$ for $x \neq 0$, and $E_{0}=Z / 2 Z$. Thus $B$ is not a strongly separable $D$. algebra, but $p: Y \rightarrow X$ is open. (It is a locally strongly separable algebra.)

Example 4.4. Let $T$ be the ring of continuous functions $f$ from $X$ to $R$ (the latter with the discrete topology) such that $f(0)$ is in $Q$. Then $X(T)=X$ and $T_{x}=R$ if $x \neq 0$ and $T_{0}=Q$. Let $A=T[x] / x^{2}+2$ and let $B=T[x] / x^{2}+3$. $A$ and $B$ are locally connected weakly Galois $T$-algebras. Now for $x$ in $X$, $\left(A \otimes_{T} B\right)_{x}=C \times C$ if $x \neq 0$ while $\left(A \otimes_{T} B\right)_{0}=Q(\sqrt{ }-2, \sqrt{ } 3)$. Thus if $S$ is a strongly separable $T$-algebra and $f: A \otimes_{T} B \rightarrow S$ a surjection, then $f_{0}$ is an isomorphism and hence (since $f$ is $T$-split) $f$ is an isomorphism on a neighborhood of 0 . But then $S$ is not locally connected. It follows that there is no locally connected locally strongly separable $T$-algebra which contains homomorphic images of both $A$ and $B$.

Example 4.5. Let $A$ be the ring of [AK, p. 477]; $A$ is a subring of the ring $B$ of all GF(4)-valued functions on a space $X$ ( $X$ has a homeomorphism $\theta$ with $\theta^{2}=1$ but $\theta$ has no fundamental domain) invariant under the ring automorphism $g$ of $B$ where $g(f)(x)=f(\theta(x))^{2}$. B is a strongly separable extension of $A$ by [CHR, 1.2(f), p. 4]. If $A$ had a separable closure $C$, the homomorphism $B \rightarrow C$ would induce a section $X(C)=X(A) \rightarrow X$ whose image is a fundamental domain for $\theta$ on $X$. Thus $A$ has no separable closure.

\section{REFERENCES}

[B]. H. Bass, Lectures on topics in algebraic K-theory, Tata Institute of Fundamental Research, Bombay, 1967.

[DI]. F. DeMeyer and E. Ingraham, Separable algebras over commutative rings, Lecture Notes in Math., no. 181, Springer-Verlag, New York, 1971. 
[G]. P. Gabriel, Construction de préschémas quotient. Schémas en groupes, Sém. Géométrie Algébrique, Inst. Hautes Études Sci., 1963/64, fasc. 2a, Exposé 5, Inst. Ha utes Études Sci., Paris, 1969. MR 41 \#1749.

[J]. G. Janusz, Separable algebras over commutative rings, Trans. Amer. Math. Soc. 122 (1966), 461-479. MR $35 \# 1585$.

[M1]. A. Magid, Galois groupoids, J. Algebra 18 (1971), 89-102.

[M2]. - Locally Galois algebras, Pacific J. Math. 33 (1970), 707-724. MR $41 \# 8405$.

[M3]. - Pierce's representation and separable algebras, Illinois J. Math. 15 (1971), 114-121.

[VZ1]. O. E. Villamayor and D. Zelinsky, Galois theory for rings with finitely many idempotents, Nagoya Math. J. 27 (1966), 721-731. MR 34 \#5880.

[VZ2]. , Galois theory with infinitely many idempotents, Nagoya Math. J. 35 (1969), 83-98. MR 39 \#5555.

[EW]. S. Endo and Y. Wata nabe, On separable algebras over a commutative ring, Osaka J. Math. 4 (1967), 233-242. MR 37 \#2796.

[P]. R. Pierce, Modules over commutative regular rings, Mem. Amer. Math. Soc. No. 70 (1967). MR $36 \# 151$.

[W]. A. Wilansky, Topology for analysis, Ginn, Waltham, Mass., 1970.

[D]. De Meyer, Separable polynomials over a commutative ring, Rocky Mt. J. Math. 2 (1972), 299-310.

[AK]. R. F. Arens and I. Kaplansky, Topological representation of algebras, Trans. Amer. Math. Soc. 63 (1948), 457-481. MR 10, 7.

[CHR]. S. U. Chase, D. K. Harrison and A. Rosenberg, Galois theory and Galois cohomology of commutative rings, Mem. Amer. Math. Soc. No. 52 (1965), 15-33. MR MR 33 \#4118.

DEPARTMENT OF MATHEMATICS, COLUMBIA UNIVERSITY, NEW YORK, NEW YORK 10027 